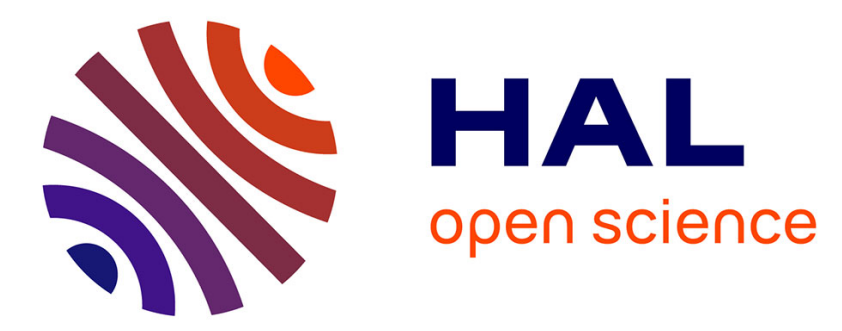

\title{
Le traitement des noms composés par les grammairiens syriaques \\ Margherita Farina
}

\section{To cite this version:}

Margherita Farina. Le traitement des noms composés par les grammairiens syriaques. Semitica et Classica, 2020, 12, pp.209-223. 10.1484/J.SEC.5.119659 . hal-02377269

\section{HAL Id: hal-02377269 \\ https://hal.science/hal-02377269}

Submitted on 7 Mar 2020

HAL is a multi-disciplinary open access archive for the deposit and dissemination of scientific research documents, whether they are published or not. The documents may come from teaching and research institutions in France or abroad, or from public or private research centers.
L'archive ouverte pluridisciplinaire HAL, est destinée au dépôt et à la diffusion de documents scientifiques de niveau recherche, publiés ou non, émanant des établissements d'enseignement et de recherche français ou étrangers, des laboratoires publics ou privés. 


\section{Margherita Farina Le traitement des noms composés par les grammairiens syriaques*}

\section{1/ LES SOURCES GRECQUES DE LA TRADITION GRAMMATICALE SYRIAQUE}

Le syriaque est un dialecte de l'araméen oriental documenté à partir du $\mathrm{II}^{\mathrm{e}}$ siècle de notre ère dans la région d'Édesse (moderne Urfa, Turquie sud orientale). En tant que langue liturgique et littéraire des communautés chrétiennes d'Orient, de l'Antiquité tardive jusqu'à l'époque contemporaine, il a connu une vaste diffusion du Proche- et Moyen-Orient jusqu'à l'Asie centrale à l'Inde et à la Chine ${ }^{1}$.

La culture littéraire syriaque a hérité de l'hellénisme diffusé au Proche-Orient pendant l'époque gréco-romaine. La pénétration du grec dans les dialectes araméens, déjà entamée dans les deux derniers siècles avant notre ère, concerne en milieu syriaque tous les niveaux de la culture, en acquérant une dimension plus proprement littéraire. La littérature syriaque connaît ainsi, entre le $\mathrm{V}^{\mathrm{e}}$ et le $\mathrm{IX}^{\mathrm{e}}$ siècle, plusieurs vagues de traductions des œuvres philosophiques et scientifiques de l'Antiquité classique ${ }^{2}$.

De même, les premiers textes syriaques à caractère linguistique, dans les domaines de la grammaire et de la logique, sont produits à partir $\mathrm{du} \mathrm{VI}^{\mathrm{e}}$ siècle de notre ère, sous la forme de traductions des textes grecs. Entre le $\mathrm{VI}^{\mathrm{e}}$ et $\mathrm{VIII}^{\mathrm{e}}$ siècle sont réalisées deux traductions du Peri hermeneias d'Aristote ${ }^{3}$ ainsi qu'une traduction de la Téchnè

\footnotetext{
* Nous avons choisi de translittérer tous les mots syriaques cités dans cet article, en tâchant d'en reproduire l'orthographe et d'en rendre bien lisible la structure morphologique aussi aux non-syriacisants. Ne s'agissant pas d'une transcription, nous ne faisons pas de distinction entre les prononciations orientale et occidentale du syriaque, notamment en ce qui concerne le signe $\bar{a}$, qui transcrit la marque vocalique désignée comme $z q \bar{a} p h \bar{a}$, quelle que soit sa prononciation dans les deux traditions. Les signes $\bar{e}, \bar{l}, \bar{o}, \bar{u}$ indiquent des voyelles notées dans l'orthographe syriaque par des matres lectionis. L'auteur remercie les relecteurs anonymes pour leurs corrections et suggestions précieuses.

1. Pour un aperçu sur la littérature et la culture syriaques, voir Briquel-Chatonnet \& Debié 2017.

2. Sur les mouvements de traduction du grec en syriaque, voir BROCK 2007, DACCACHE 2018, URL = https://www. encyclopedie-humanisme.com/Traduction-en-syriaque; sur les techniques de traduction, voir BROCK 1977, 1979 et 2004.

3. Hugonnard-Roche 2004 et 2018. Pour la place de l'enseignement grammatical dans l'éducation au Proche-Orient dans l'Antiquité tardive, voir WATT 1993.
}

grammatikè du Pseudo-Denys le Thrace ${ }^{4}$. Cette dernière représente aussi la première description grammaticale de la langue syriaque dont nous ayons connaissance.

Un autre modèle grammatical majeur est représenté par les Canones de Théodose d'Alexandrie ( $\mathrm{IV}^{\mathrm{e}}-\mathrm{V}^{\mathrm{e}}$ siècle), assimilés et adaptés au syriaque dans le Tūrās mamllā (« la correction de la langue ») de Jacques d'Édesse (m. 708), dont seulement quelques fragments survivent ${ }^{5}$. D'ailleurs, nous pouvons aussi mesurer l'influence de la linguistique grecque sur ce savant syriaque par d'autres ouvrages qui nous sont conservés, notamment un traité sur l'orthographe et un petit traité grammatical sur l'accentuation ${ }^{6}$, ainsi que par ses innombrables traductions d'œuvres grecques dans divers domaines du savoir ${ }^{7}$. C'est donc à partir d'un noyau fondamental, constitué par les traductions et adaptations de la Téchnè grammatikè et de ses scholia, des Canones, du Peri hermeneias et de ses commentaires (surtout le commentaire de Proba, au $\mathrm{VI}^{\mathrm{e}}$ siècle ${ }^{8}$ ) que se développent les premières réflexions sur la langue syriaque.

En même temps, le modèle grec n'est pas reçu et reproduit de façon passive. Au contraire, l'effort progressif de compréhension et d'adaptation, en faisant émerger les différences structurelles entre langue source et langue cible, nourrit la conscience métalinguistique des grammairiens syriaques, qui parviennent à élaborer de nouvelles théories et à découvrir des catégories indépendantes.

Le sujet abordé dans cet article - les noms composés (syriaque $\check{s} m \bar{a} h \bar{e} m r a k b \bar{e}$ ) - touche au vif des relations linguistiques gréco-syriaques. Il s'agit à la fois de l'intégration au sein du syriaque de mots d'origine grecque à la morphologie spécifique (mots grecs comme $\theta \varepsilon 0 \lambda o \gamma i \alpha$, $\mu \eta \tau \rho \delta ́ \pi \sigma \lambda \iota \varsigma, \varphi \iota \lambda о \sigma o \varphi i ́ \alpha$, etc., aussi bien que leurs correspondants syriaques) et de l'assimilation de la théorie grammaticale qui les décrit. Nous tâcherons de montrer comment, par l'introduction d'une catégorie grammaticale, apparemment peu adaptée à la structure morphosyntaxique

4. TALmon 2000 et Contini 2001. Le texte de la Téchnè syriaque a été édité par Merx 1889 , p. $50^{*}-72 *$, qui a donné aussi une comparaison critique avec le texte grec (p. 9-28). Une liste des manuscrits syriaques qui conservent la traduction de Huzāyā se trouve dans Farina 2018a, p. 250.

5. Éd. Wright 1871. Voir Farina 2018b, TALMON 2008.

6. Cf. FARINA 2018 , p. 169-179.

7. Cf. TALMON 2008.

8. Éd. HofFMANN 1869, voir sur cet auteur et sur son commentaire HugOnNARD-Roche 2004. 
d'une langue sémitique, les grammairiens syriaques créent une réflexion métalinguistique originale, en parvenant à déceler des éléments propres à leur langue.

\section{2/ LES NOMS COMPOSÉS EN SYRIAQUE}

Comme plusieurs langues indo-européennes, le grec se caractérise par la possibilité de former des noms composés. Deux noms peuvent être combinés dans une structure où le thème du premier (radical + voyelle thématique) est attaché au deuxième nom. Les deux noms forment ainsi un nouveau mot à la structure stable, dont le deuxième membre constitue la tête du composé (il porte les marques de la déclinaison et détermine l'accord). La relation de dépendance entre les deux noms possède un spectre sémantique large qui va de la juxtaposition ( $\rho \rho \alpha \gamma \varepsilon ́ \lambda \alpha \varphi \circ \varsigma$ « chèvre-cerf », un animal mi-chèvre et mi-

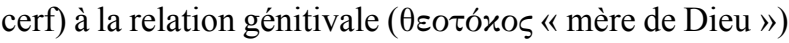

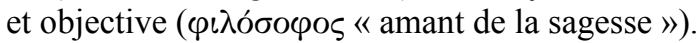

La composition nominale est un procédé relativement rare en syriaque, et en général dans les langues sémitiques. ${ }^{9}$ Dans les dialectes plus anciens on trouve des anthroponymes et des toponymes sous forme de microphrases, composés soit de deux (ou plus) éléments nominaux soit d'un élément verbal et un élément nominal : akkadien Šamašrabi « (le dieu) Šamaš (est) grand » ou Iddin-Sîn «(le dieu) Sin a donné $»^{10}$. Il existe aussi un type de composé anthroponymique où, deux éléments nominaux sont en relation génitivale (voir infra), comme Itrūtt-[Nom divin] «Richesse de [Nom divin] » ${ }^{11}$. Les mêmes structures nominales se retrouvent en ougaritique : l'anthroponyme $\bar{I} l \bar{u}$-milku « le dieu est roi » ou le toponyme Ili 'ištama « « le dieu a écouté »; en hébreu biblique : 'Abimelek « mon père est roi » ou bien 'Ahazyāhū « (le dieu) Yahū a tenu », etc. Il s'agit pour la plupart de noms théophores, c'est-à-dire des microphrases contenant un nom divin. Le syriaque a retenu ce type de noms propres, comme nous le verrons dans la suite, tant à travers la tradition biblique que par de nouvelles formations d'origine chrétienne (parfois sur modèle persan, v. infra), comme Māran'ammeh « Notre Seigneur (est) avec lui » pour le premier type ou, pour le en deux mots ? deuxième type, $\bar{I} \bar{s}^{\llcorner} \bar{u}^{`}$ yahb « Jésus a donné ».

En dehors de l'onomastique, les deux principales stratégies morphosyntaxiques que le syriaque connaît pour associer deux substantifs dans une relation de dépendance sont :

9. GENSLER 2011, p. 287-288.

10. HuEHNERGARD 2005, $\S 13.1$. Un aperçu très concis des phases plus anciennes de l'onomastique akkadienne ancienne se trouve dans CATAGNOTI 2005.

11. CATAgnoti 2005, p. 150. a/ La relation génitivale (ou annexion) entre un nomen regens à la morpho-phonologie réduite (état construit) et un nomen rectus en forme pleine (état absolu rectum? ou emphatique) ${ }^{12}$.

Par exemple, étant donné les substantifs $b r \bar{a} «$ fils » et 'nāš $\bar{a}$ « homme », on peut avoir l'annexion bar 'nāša « fils de l'homme », ensuite stabilisée dans le composé barnāšă, qui signifie tout simplement « homme ». Ou encore, à partir des substantifs bartā « fille » et qālā « voix », on a le composé brat qālā (ou même parfois brat qālā) « fille de la voix », qui indique le « mot» ou le « son » doué d'un signifié. Certains composés de ce type ont connu un processus d'univerbation et sont écrits communément comme un seul mot: šetestā «fondement» de 'eštā « fonds » (documenté uniquement dans ce composé) + 'estā « mur », rabbaytā « intendant » de rabā « chef »+ baytā $\ll$ maison $»^{13}$.

b/ Un syntagme relatif, où deux noms à la morphophonologie pleine sont associés à travers la particule relative $d$ -

Par exemple, avec les substantifs brā « fils » et 'alāhā « Dieu », on peut avoir le syntagme relatif $b r \bar{a} d$-'alāhā « fils de Dieu ».

Par ailleurs, le syriaque comme d'autres dialectes de l'araméen plus anciens ou contemporains (araméen d'empire, araméen judéo-babylonien) reçoit une grande quantité de noms composés empruntés au grec (GR) ou au moyen-perse $(\mathrm{MP})^{14}$. De tels composés sont soit intégrés dans la langue dans leur forme originelle, parfois avec quelques adaptations phonologiques ou purement graphiques, soit traduits sous forme de calques. Dans ce deuxième cas, les calques tâchent de reproduire aussi la structure morphologique du composé, en ayant recours à l'état construit (type a/ ci-dessus), dont ils forcent et élargissent le potentiel sémantique. Voici quelques exemples :

12. Le nom syriaque peut se présenter sous trois formes différentes, traditionnellement appelées " états » : l'état absolu est la forme de base, utilisée dans la prédication nominale (e.g. $k t a \bar{b}$ « livre »), l'état emphatique, forme augmentée par la marque $-\bar{a}$, à l'origine marque de détermination (e.g. ktā $b \bar{a}$ « le livre » ou bien " un livre ») et l'état construit, forme utilisée dans l'annexion (e.g. ktāa « livre de...»). Bien qu'au masculin singulier l'état construit et l'état absolu soient identiques, des différences dans le paradigme témoignent de leur différenciation originelle.

13. Duval 1881, p. 243-244, Nöldeke 1898, § 141. Ce phénomène est bien documenté aussi dans des dialectes néoaraméens plus ou moins proches du syriaque : CiancAgLinI \& AlFiERI 2013, p. 110, 119-120.

14. Schall 1960, Ciancaglini 2008 et 2010, Ciancaglini \& AlFIERI 2013. 


\begin{tabular}{|c|c|c|c|}
\hline SOURCE & $\begin{array}{l}\text { EMPRUNT } \\
\text { SYRIAQUE }\end{array}$ & $\begin{array}{c}\text { CALQUE / } \\
\text { TRADUCTION SYRIAQUE }\end{array}$ & TRADUCTION \\
\hline GR $\mu \eta \tau \rho o ́ \pi o \lambda ı s$ & metrōpōlīs & & métropole \\
\hline GR $\pi \alpha \tau \rho \iota \alpha \rho \chi \eta \varsigma$ & pațrīarkā & & patriarche \\
\hline 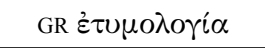 & 'etūmōlōgīa & & étymologie \\
\hline GR $\pi 0 \lambda v \pi o ́ \delta \iota \nu^{(1)}$ & polūpodyōn & saggī reglā & « beaucoup de pieds », polypode \\
\hline 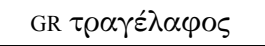 & & ‘nez 'aylā & « chèvre de cerf $»>$ hircocerf (mais aussi une sorte de gazelle ${ }^{(2)}$ ) \\
\hline GR $\alpha \rho \nu o ́ \gamma \lambda \omega \sigma \sigma o \nu^{(3)}$ & 'rnglswn & lešān 'emrā & « langue d'agneau » (nom du plantain) \\
\hline GR $\pi \alpha \nu \tau O \varkappa \rho \alpha ́ \tau \omega \rho^{(4)}$ & & 'aḥid kul & « régisseur de tout $»>$ tout-puissant \\
\hline GR oivorótทs & & šătē ḥamrā & buveur de vin \\
\hline MP šāhānšāa $h^{(5)}$ & & mlek malkē & roi des rois \\
\hline MP $x a r b u z^{(6)}$ & karbūz & 'nez ḥemrā & « chèvre de l'âne » > antilope \\
\hline \multicolumn{4}{|c|}{ 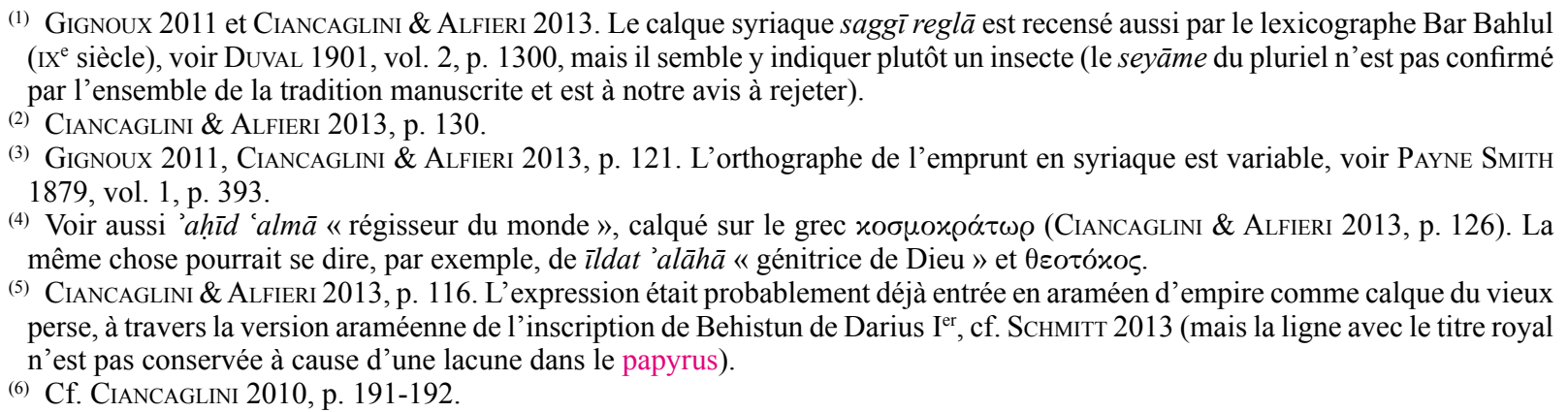 } \\
\hline
\end{tabular}

Tous les calques présentés dans ce tableau ont la forme décrite en a/ ci-dessus : une annexion où le premier terme est à l'état construit et le deuxième en forme pleine.

Si des calques comme lešān 'emrā ou mlek malkē sont tout à fait transparents et ne posent aucun problème par rapport à la syntaxe nominale syriaque, d'autres, comme 'nez 'aylā ou 'nez hemrā, forcent la relation de dépendance que l'état construit implique : 'nez 'aylā ne signifie pas « chèvre de cerf » mais plutôt chèvre-cerf, impliquant une relation «chèvre »+ « cerf ». Encore, 'aḥīd kul et šâtē hamrā impliquent une relation de type objectif entre le nomen regens, dans ces deux cas un participe ('ahîd « qui tient », šâte « qui boit ») et le nomen rectum (kul « tout», hamrā « vin »), qui a comme référent l'objet de l'action exprimée par le participe ${ }^{15}$.

15. Une relation semblable est exprimée par des états construits comme 'ūhdān māreh « la mémoire de son maitre » ou šqāl ta $n \bar{a}$ 《 soulèvement du poids $>$ diligence », où le premier membre est néanmoins un substantif(NöLDEKE 1898, § 205).

\section{3/ LES SOURCES GRECQUES SUR LES NOMS COMPOSÉS ET LEURS ADAPTATIONS SYRIAQUES}

Les sources grecques dont les auteurs syriaques se sont inspirés pour la description des noms composés sont essentiellement la Téchnè du Pseudo-Denys le Thrace traduite au $\mathrm{VI}^{\mathrm{e}}$ siècle; le Peri hermeneias (deux traductions entre le $\mathrm{VI}^{\mathrm{e}}$ et le $\mathrm{VIII}^{\mathrm{e}}$ siècle) et le commentaire de Proba; les Canones de Théodose, adaptés en syriaque par Jacques d'Édesse à la fin du viI ${ }^{\mathrm{e}}$ siècle dans sa grammaire syriaque. Nous avons malheureusement perdu la section sur les canons des noms composés de cette œuvre, mais, comme nous le verrons $(\$ 4.1)$, il nous en reste une trace indirecte chez Jean le Stylite.

\section{1/ La Téchnè syriaque}

La Téchnè grammatikè traite des noms composés dans

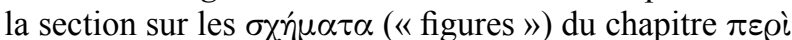
óvó $\mu \alpha \tau O \varsigma^{16}$. Trois figures du nom y sont distinguées :

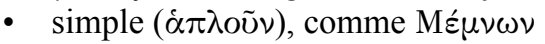

- composé ( $\sigma u ́ \nu \theta \varepsilon \tau \sigma \nu)$, comme 'A $\gamma \alpha \mu \varepsilon \dot{\varepsilon} \mu \omega \nu$

16. Texte et traduction française dans LALLOT 1998, p. 53, 12 C. 
- dérivé de composé ( $\pi \alpha \rho \alpha \sigma u ́ \nu \theta \varepsilon \tau o \nu)$, comme

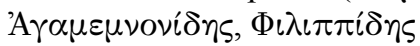

Les composés sont ensuite divisés en quatre variétés, selon leur structure morphologique et la forme des deux membres dont ils sont constitués :

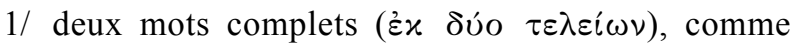

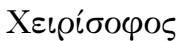

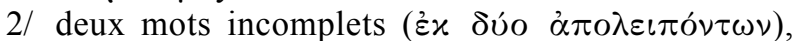
comme $\Sigma o \varphi 0 x \lambda \tilde{\eta} \varsigma$

3/ un mot incomplet et un complet ( $\dot{\xi} \xi \dot{\alpha} \pi 0 \lambda \varepsilon i \pi \sigma o \nu \tau o \varsigma$

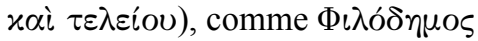

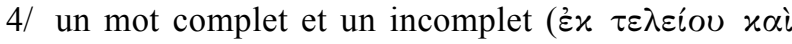

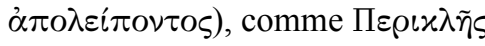

Voici comment le texte grec a été transposé et adapté en syriaque ${ }^{17}$ :

Les figures ('eskimēe du nom sont au nombre de trois: simple (pšiț $\bar{a})$, composé (mrakbā) et plus que composé (yatīr men mrakbā). Le simple est, par exemple, 'ab [père], le composé est 'Abrām [Abram] et le plus que composé est 'Abrāhām [Abraham]. Il y a trois variétés de composés : [1.] ceux qui sont composés de deux [noms] complets (mšamlyē), de telle sorte qu'ils ne deviennent qu'un seul [aucun exemple]; [2.] ceux qui sont composés de deux parties incomplètes (hasīrātẫ), comme Mū̌še [Moïse]; [3.] ceux qui sont composés d'une partie incomplète (hasìrtā) et une complète (mšalmānìtā), comme mlek malkē [roi des rois]; [4.] ceux qui sont composés d'une partie complète et une incomplète, comme bar-dādeh [lit. fils de son ami, ou de son oncle $>$ cousin $]^{18}$.

Au point de vue de la terminologie, nous observons les correspondances suivantes :

- gr. $\dot{\alpha} \pi \lambda o \tilde{v} \nu$ - syr. pšît $\bar{a}$ « droit, simple, commun », participe du verbe $p s ̌ a t ~ «$ rendre droit, aplanir »;

- gr. $\sigma u ́ v \theta \varepsilon \tau o \nu$ - syr. $m r a k b \bar{a}$ « composé », participe du verbe rakkeb « construire, composer »;

- gr. $\pi \alpha \rho \alpha \sigma u ́ \nu \theta \varepsilon \tau o \nu$ - syr. yatīr men mrakbā «plus que composé »;

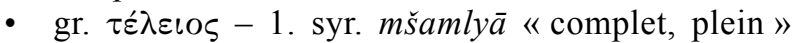
participe $\check{s} a f^{‘} e l$ du verbe $m l \bar{a}$ « remplir »;2. mšalmānītā nom dérivé de la racine šlm « achever, compléter»; l'oscillation entre les deux racines se reflète dans toute la tradition suivante;

- gr. $\alpha \pi 0 \lambda \varepsilon i \pi o \nu$ - syr. hasīitā participe du verbe hsar «manquer de, être insuffisant ».

17. Texte syriaque selon l'édition de Merx 1889, p. 54*.

18. Certains manuscrits ont les variantes Bar-Hādād, nom propre, « fils de Hadad » (cf. PAyne Smith 1879, vol. 1, p. 974) ou même $b z$ hdd (?), cf. MerX 1889, p. 54*, 1. 16.
$\mathrm{Si}$, d'un côté, le texte syriaque suit de très près la systématisation de son modèle, en en reprenant le lien entre la catégorie des composés et l'étymologie des noms propres, ainsi que toutes les quatre sous-catégories des composés, d'un autre côté le choix des exemples s'éloigne sensiblement du texte grec. En dépit du grand nombre de noms composés grecs empruntés par le syriaque, le traducteur de la Téchnè préfère se servir d'exemples syriaques, malgré leur obscurité substantielle et la correspondance imparfaite avec la catégorie morphologique grecque.

La première triade 'ab-'Abrām-'Abrāhām renvoie à Gn 17,5 «On ne t'appellera plus Abram; mais ton nom sera Abraham, car je te rends père d'une multitude de nations. » Le passage hébraïque de la Genèse repose sur une étymologie des noms 'Abrām ('ab «père »+ rām « élevé ») et 'Abrāhām ('ab « père » + hām/hāmōn « multitude »? ${ }^{19}$ ) et fait du nom du patriarche le composé par excellence, ainsi qu'un modèle structurel dans l'onomastique sémitique.

L'exemple de la classe [2] est lui aussi d'origine biblique. Dans la Bible, la parétymologie du nom de Moïse (hébr. mošeh) le fait dériver du verbe hébreu $m a \bar{a} s \bar{a} h$ « sauver », Moïse étant celui qui a été sauvé des eaux (cf. Ex 2,10 : «Elle lui donna le nom de Moïse, car, ditelle, je l'ai retiré des eaux »). La traduction syriaque du verset (version Pešitțā) récite men mayā šlìteh « de l'eau je l'ai tiré ». Le remplacement de la racine hébraïque $m s ̌ h$ par la racine araméenne šly a amené Huzāyā à reformuler l'étymologie du nom comme composé des mots maya « eau» et šly «tirer de l'eau», mais tous les deux « incomplets », l'un ayant donné au mot son $m$ - initial et l'autre le $\check{s}$ -

Le recours à l'étymologie des noms de deux figures fondamentales de la tradition judéo-chrétienne semble indiquer une source dans le milieu exégétique et dans la pratique du commentaire.

Le composé mlek malkē « roi des rois » pourrait aussi avoir une ascendance biblique, dans ce cas du Nouveau

19. L'étymologie biblique est interprétée par les grammairiens syriaques comme un nouveau composé du mot ' $a b$ « père », qui présuppose ensuite un substantif * rāhām, issu d'une racine * rhm non autrement documentée. En supposant que le mot hébraïque hām/hāmōn «multitude " soit à l'origine de l'étymologie de la Genèse, on peut supposer une réinterprétation du composé 'abir « chef » + hām/hāmōn (nous remercions le relecteur anonyme de nous avoir indiqué cette interprétation). Sur la base de la mention d'une tribu des Raham dans une stèle de Sethi I ${ }^{\text {er }}$ à Beth Shehan («the Asiatics of Rehem », PRITCHARD 1969, p. 255-256), Liverani (LiverANi 2003, p. 30) suppose, par ailleurs, l'existence d'une tribu des *Banu Rāhām, ayant comme éponyme un *Abu-Raham, ce qui supporterait la segmentation biblique du composé. dire qui c'est?

Ou développer la note 4 et $\mathrm{y}$ renvoyer? 


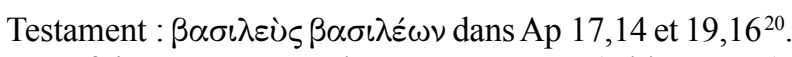
Toutefois, comme nous l'avons vu au $\S 2$ (tableau, n. 5), l'expression est plus largement répandue en syriaque à travers un calque du vieux perse en araméen d'empire.

Enfin, l'exemple de la quatrième classe se trouve dans la mauvaise catégorie, que l'on choisisse la forme Barhādād ou bien Bar-dādeh, car il s'agit d'un état construit tout comme mlek malkē. Bar-dädeh pourrait tout de même être considéré comme un exemple du plus que composé, puisqu'il est constitué de trois membres : bar- «fils de » $+d \bar{a} d$ - « ami de/oncle de »-eh « lui ».

Tous ces exemples révèlent une interprétation floue des concepts de « complet» et « incomplet ». Si dans le cas de mlek malkē on pourrait supposer que le traducteur leur donne un contenu morphologique et qu'il s'en sert pour désigner respectivement l'état construit (forme " abrégée ») et l'état emphatique (forme avec la marque de détermination $-\bar{e}$, voir supra $\mathrm{n}$. 9), il n'en est pas ainsi pour $M \bar{u} \bar{s} \bar{e}$, analysé comme composé de morceaux de mots recoupés au petit bonheur.

\section{2/ Les versions syriaques du Peri hermeneias et le commentaire de Proba}

Dans les premiers paragraphes du Peri hermeneias, Aristote introduit une distinction entre nom simple

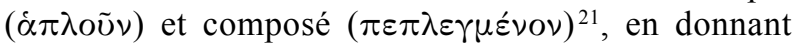
pour cette deuxième catégorie les exemples suivants :

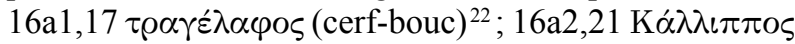
(Callippos, nom propre); 16a2,27 $\dot{\varepsilon} \pi \alpha x \tau \rho o x \varepsilon \dot{\varepsilon} \eta \varsigma$ (vaisseau pirate) ${ }^{23}$.

Les traductions syriaques du Peri hermeneias et le commentaire de Proba font les choix suivants ${ }^{24}$ :

20. Il est intéressant d'observer que la Peshitțā du Nouveau Testament traduit avec mlek malkē (composé de type $a$ ) en Ap 17, 14, mais avec malkā $d$-malkē (composé de type $b$ ) en Ap 19,16.

21. On trouve une terminologie différente dans la Poétique

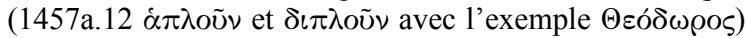
et dans la Rhétorique (1406a.29 $\alpha \pi \lambda$ oũv et $\dot{\alpha} \nu \tau i ́ \mu \mu о \varsigma)$.

22. Pour l'identification de cet animal et sa présence dans la tradition littéraire grecque, voir MONTANARI 1984-1988, p. 72-74.

23. Qu'Aristote utilise Kó $\lambda \lambda \iota \pi \pi$ ' comme exemple d'un nom composé demeure incertain (cf. Belardi 1985, p. 111, MONTANARI 1984-1988, p. 97-103). Nous ne rentrerons pas dans ce débat, en nous limitant à observer le comportement des traducteurs et commentateurs syriaques qui, en suivant les interprètes grecs anciens comme Ammonius et Stéphanos, le

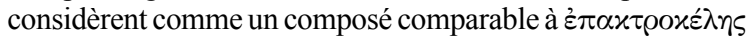
(voir MONTANARi 1984-1988, p. 98).

24. Les trois textes sont édités dans Hoffmann 1869. Une description détaillée des sources et une analyse critique se trouvent dans HugONNARD-ROCHE 2004, p. 57-77.

\begin{tabular}{|c|c|c|}
\hline $\begin{array}{c}\text { TRADUCTION } \\
\text { ANONYME VI }{ }^{\mathrm{e}} \text { SIĖCLE }\end{array}$ & $\begin{array}{l}\text { COMmentaire DE } \\
\text { Proba VI }{ }^{\mathrm{e}} \text { SIĖCle }\end{array}$ & $\begin{array}{c}\text { Traduction } \\
\text { PAR GeORGE DES } \\
\text { ARABES VIII }{ }^{\mathrm{e}} \text { SIĖCLE }\end{array}$ \\
\hline 16a1,17 'nez 'aylā & 'nez 'aylā & 'nez 'aylā \\
\hline 16a2,21 qlypws & ‘ūqbrāa « souris » & 'aḥūhā-d-'emmeh \\
\hline 16a2,27 'pwp'qrtqlys & - & $b l m w d d^{(1)}$ \\
\hline \multicolumn{3}{|c|}{ 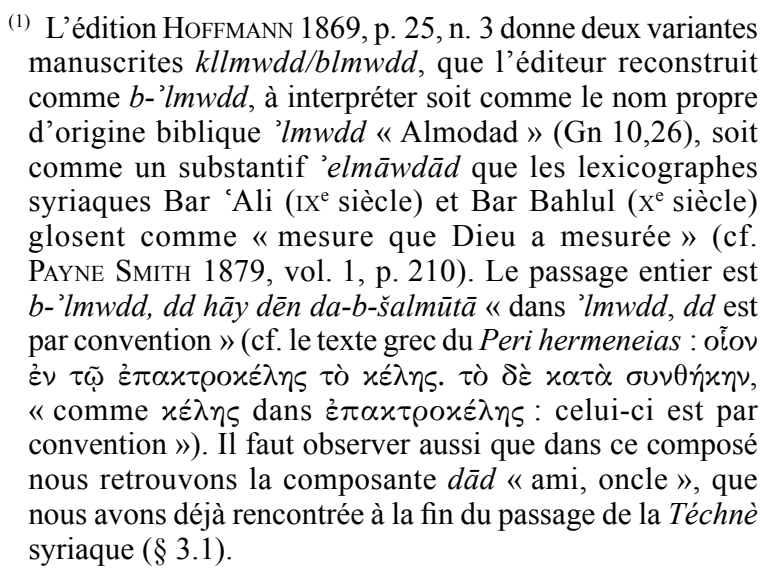 } \\
\hline
\end{tabular}

Toutes les versions et le commentaire concordent dans la traduction de $\tau \rho \alpha \gamma \varepsilon ́ \lambda \alpha \varphi \circ \varsigma$ par 'nez 'aylä « chèvre de cerf », composé de type a/ (relation génitivale) qui devait donc être une traduction établie par la tradition.

Pour Kó $\lambda \lambda \iota \pi \pi \circ \varsigma$ nous avons d'abord, dans la traduction $\mathrm{du} \mathrm{vI} \mathrm{e}^{\mathrm{e}}$ siècle, la simple translitération du mot grec, suivie par la décomposition qlwsypws. Il en va de

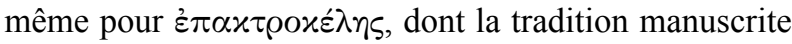
nous restitue une translitération un peu imprécise.

Proba substitue à cet exemple une adaptation au syriaque de l'exemple qu'Aristote introduit au chapitre IV $(16 \mathrm{~b}, 31-32): \mu \tilde{\nu} \varsigma$ « souris » ne peut pas être analysé en $\mu+v \varsigma$ (même si le mot $\tilde{\zeta} \varsigma$ " porc » existe). Le commentateur adapte cet exemple en utilisant le mot syriaque ' $\bar{u} q b r \bar{a}$ « souris », dont il n'est pas possible d'extraire la souspartie signifiante $b r \bar{a}$ « fils ».

Georges des Arabes, par contre, choisit de remplacer le nom propre composé du grec par un nom propre syriaque qu'il considère comme ayant une structure analogue : 'Ahūhä-d-'emmeh littéralement «le frère de sa mère ", un composé du type b/ avec particule relative $d$-, un nom syriaque très répandu, le plus souvent contracté en 'Ahüdemmeh.

Comme nous le verrons par la suite, certains des exemples du Peri hermeneias, dans ses différentes versions, ont pénétré aussi dans les textes.

Deux traductions arabes du Peri hermeneias sont issues d'un milieu chrétien syro-arabe. La première, anonyme mais probablement attribuable à Hunayn ibn Isḥaq (808- 
873) ou à son fils Isḥaq ibn Ḥunayn $(830-910)^{25}$, la deuxième par le même Isḥaq ibn Hunayn ${ }^{26}$. Ces versions suivent, dans le traitement des composés, des parcours qui se rapprochent parfois des versions syriaques. Le

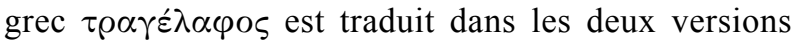
par le composé 'anz 'iyyal " chèvre-cerf », équivalent étymologique du syriaque 'nez 'aylä ${ }^{27}$. Il est probable que ce composé d'origine perse était devenu commun tant en arabe qu'en syriaque. Pour Kó $\lambda \lambda \iota \imath \pi \pi \circ \varsigma$, la version anonyme ${ }^{28}$ et celle d'Ishaq Ibn Hunayn ${ }^{29}$ donnent la translitération qālibus/qalibus, analysée en deux membres $q \bar{a} l \bar{u} s \bar{i} b u \bar{s} / q \bar{a} l \bar{u} s$ ibus. Isḥaq glose ensuite avec l'arabe faras färih (« cheval gracieux »). Le passage concernant

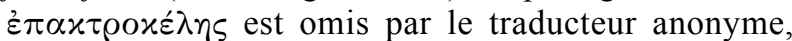
alors qu'Ishaq lui substitue l'emprunt grec fìlūsū $\bar{f} \bar{u} s$, qu'il glose ensuite comme mu'tir 'al-hikma «sectateur de la sagesse $»^{30}$. L'arabe limite donc le recours aux composés de type a/ (état construit) au seul cas de 'anz 'iyyal, en utilisant dans les autres cas des emprunts grecs accompagnés de gloses littérales aux membres des composés.

\section{4/ LE TRAITEMENT DES COMPOSÉS PAR LES GRAMMAIRIENS SYRIAQUES APRÈS LE VI ${ }^{\mathrm{e}}$ SIĖCLE}

\section{1/ Jean le Stylite et la réélaboration de l'héritage grec}

Il ne nous reste que quelques fragments de la grammaire syriaque composée par Jacques d’Édesse (m. 708), en bonne partie sur le modèle des Canones de Théodose. Nous n'avons donc aucun témoignage direct de sa pensée sur les noms composés. Toutefois, Axel Moberg a cru reconnaître une trace évidente des théories de Jacques dans la grammaire de Jean le Stylite (en syriaque Yuhannān Esțūnāyā), auteur qu'il situe dans la première moitié du IX ${ }^{e}$ siècle $^{31}$. Nous n'avons pas pu consulter les deux seules copies existantes de ce texte, qui se trouvent dans une région du Moyen-Orient d'accès difficile à

25. Texte incomplet, édité dans HoffMann 1869, p. 55-61. Cf. HugONNARD-ROCHE 2004, p. 69-71. Sur ces deux auteurs voir, respectivement, ButTs 2011 et STROHMAIER 2010, consulté en ligne le 3 mai 2019. Sur le mouvement de traduction qu'ils ont animé voir GuTAS 1998. Voir aussi, pour une réévaluation de l'apport syriaque, BRock 1991, WATT 2004.

26. Texte édité par POLLAK 1913.

27. Hoffmann 1869, p. 56, Pollak 1913, p. 2.

28. HofFMANn 1869, p. 56.

29. Pollak 1913, p. 2-3.

30. POLLAK 1913 , p. 3, traduction et commentaire par HugONNARDROCHE 2004, p. 72.

31. Moberg 1909. L'identification du grammairien Jean le Stylite demeure très incertaine. Pour une discussion plus récente (mais pas plus avancée) voir aussi SuERMANN 2009. présent ${ }^{32}$. Néanmoins, Moberg a donné une traduction allemande de certains paragraphes de l'ouvrage, y compris de la section sur les noms composés, que nous avons ainsi la possibilité d'examiner, bien que de façon indirecte. À la

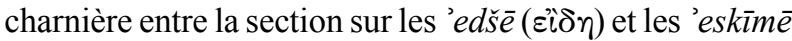
$(\sigma \chi \eta \dot{\eta} \mu \alpha \tau \alpha)^{33}$, Jean observe que :

[...] en général, tous les noms qui se trouvent dans cette langue syriaque sont simples. S'il se trouve qu'ils sont composés, ils ne respectent pas les xóvoves de la langue parce que, parmi eux, certains sont composés d'un verbe et un nom, d'autres d'un nom incomplet et d'un nom complet, d'autres d'un verbe et d'un nom incomplet, d'autres de plusieurs membres. [Formés] d'un verbe et d'un nom, il existe aussi des composés tels que $p \bar{a} l a h h^{\prime} a r^{\prime} \bar{a}$ [laboureur de la terre], šătê hamrāa [buveur de vin], d'un nom incomplet et d'un nom complet, tels que pill tayšsa [bouc-éléphant], 'nez 'aylā, mlek malkē, d'un verbe et d'un nom incomplet, tels

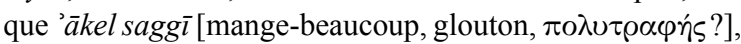
qaddīš-kul [tout-saint], 'ahīd-kul [tout-tenant, tout-puissant], et de plusieurs membres, tels que la yāda' napšeh [qui ne se connaît pas soi-même], la 'dīl b-dūbreh [irréprochable dans

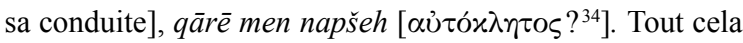
pour les 'eďse [espèces, formes] des noms ${ }^{35}$.

Par rapport à la Téchnè, nous pouvons observer une véritable révolution dans la catégorisation et dans le traitement de la catégorie des composés. Comme l'a justement observé Moberg, la référence aux canones de la langue renvoie certainement à Théodose et donc à Jacques d'Édesse ${ }^{36}$. Il est même possible d'aller plus loin et de

32. Il s'agit du manuscrit Vosté $290=$ Haddad 890 du couvent de Notre-Dame-des-Semences près d'Alqoš, un recueil de textes grammaticaux décrit dans les catalogues SCHER 1906, n. 139 (daté par Scher au XVI e siècle), Vosté 1929, n. 290, HADDAD \& ISAAQ 1988, n. 890. Toutefois Vosté affirme que le manuscrit était devenu presque illisible à son époque et ne fait plus mention du texte de Jean le Stylite. Moberg affirme avoir travaillé sur une copie qu'il avait reçue de Scher, dont nous n'avons pas pu retrouver la trace.

33. L'inversion des noms des accidents du nom 'edšè et 'eskīmē était une spécificité de Jacques d'Édesse. En la retrouvant chez Jean le Stylite, Moberg l'utilise comme argument pour établir une dépendance du deuxième par rapport au premier : Moberg 1909, p. 32.

34. L'expression, qui signifie littéralement « qui appelle par soimême », ne figure pas dans les lexiques syriaques. On pourrait supposer qu'elle traduit le composé grec $\alpha u ̛ \tau o ́ x \lambda \eta \tau o \varsigma$, bien documenté chez les pères grecs (Grégoire de Nysse, Jean Chrysostome, Cyrille d'Alexandrie, Origène, cf. TLG 2001), sur le même modèle qu' $\alpha$ $\tau o \mu \alpha \theta \dot{n} s-\bar{\imath} d \bar{u}^{\top} \bar{a}$ men napšeh « qui s'est instruit par soi-même » (cf. PAYNe SMITH 1879, vol. 2, p. 2431)

35. Traduction de l'allemand de Moberg 1909, p. 27.

36. « Le sens de l'expression devient immédiatement clair si l'on donne à ce mot le sens qu'il a dans la grammaire de Jacques d'Édesse, le même qu'avait le mot $\varkappa \alpha \nu \omega ́ \nu$ dans le modèle de il faudrait construire la phrase autrement : syntaxe incorrecte 
constater que le problème des composés traités par Jean le Stylite est qu'ils sont justement formés de plusieurs mots, alors qu'il vient d'énoncer plus haut l'équation nom syriaque $=$ simple $^{37}$. Il est peut-être possible d'y voir un écho d'une des scholies à la Téchnè : « [le composé] résulte de la réunion sous un unique accent pour s'appliquer à un unique référent de deux ou plusieurs mots pensables individuellement $»{ }^{38}$ Pour pouvoir appartenir à la classe des noms - semble nous dire Jean le Stylite - un composé doit se présenter comme un unique référent. Les composés de type a/, à l'état construit, peuvent bien constituer une seule unité phonétique (voir $\S 2$ ), mais cela n'est pas le cas des locutions plus complexes présentées à la fin du passage.

Les exemples tirés de l'onomastique, que l'on trouvait dans la Téchnè, ici ont complètement disparu, pour laisser la place à une typologie complexe de composés syntactiques.

Nous ne pouvons pas vérifier les termes syriaques employés par Jean le Stylite, mais il est clair que chez lui les notions de « complet » et « incomplet » ont acquis un sens morphologique précis et se réfèrent systématiquement à l'opposition entre état construit/absolu d'un côté et état emphatique de l'autre ${ }^{39}$. Ainsi, pĭl tayša $\bar{a}$ est composé du nom à l'état construit $p \bar{l} l$ « éléphant » et du nom à l'état emphatique tayša « bouc» (avec la marque de détermination $-\bar{a})$; par contre 'ākel saggī est composé du participe 'ākel «mangeur» et de l'adjectif saggī « nombreux » à l'état absolu.

Selon le lexique grammatical syriaque rédigé par Moberg ${ }^{40}$, le terme mšamlyā «complet » est utilisé pour indiquer l'état emphatique du nom par plusieurs grammairiens syriaques du XIII ${ }^{\mathrm{e}}$ siècle (Bar Zo ${ }^{\mathrm{c}} \mathrm{bi}$, Bar Šakko, Barhebraeus). Le même sens peut aussi être donné à son concurrent $\check{s} a l m \bar{a}$ (surtout chez Barhebraeus, sur lequel voir infra $)^{41}$.

Jacques, les Canones Alexandrini de Théodose : par exemple, šātē hamrā, considéré comme un seul mot (composé), ne peut pas être classé selon les règles courantes de formation des noms en syriaque » Moberg 1909, p. 32.

37. Nous retrouvons une démarche assez similaire dans l'ouverture du chapitre sur le nom de la grammaire d'un dialecte néo-araméen (le néo-araméen juif de Betanure) : «There are two major morphological categories of nouns, one comprising original Aramaic and Aramaicized nouns, the other comprising foreign nouns unadapted to Aramaic morphology », MUTZAFI 2008, p. 91.

38. LALlOT 1998, p. 137.

39. Pour les trois états du nom syriaque voir supra n. 12.

40. Moberg 1907-1913, vol. 2, p. 55*.

41. Moberg 1907-1913, vol. 2, p. 100*.
Jean le Stylite ${ }^{42}$ esquisse ainsi une typologie de la morphosyntaxe de la composition nominale en syriaque ${ }^{43}$, qui devient indépendante du modèle grec.

En ayant discuté les noms simples et composés au sein des 'ed $̌ \bar{s} \bar{e}$, Jean les reprend brièvement dans la section suivante, consacrée aux 'eskimme (du grec $\sigma \chi \eta \dot{\eta} \mu \alpha \tau \alpha)$, qu'il introduit par la rubrique « autrement » : « simple est, par exemple, le mot 'Abrām; composé est, par exemple, le mot 'Abrāhām, plus que composé est, par exemple, le mot bar-hānūm $»^{44}$.

\section{2/ L'approche lexicographique de David Bar Paulos}

Un petit traité sur les noms composés nous est parvenu sous le nom de David Bar Paulos, auteur syro-occidental qui vécut entre le $\mathrm{VIII}^{\mathrm{e}}$ et le $\mathrm{IX}^{\mathrm{e}}$ siècle et qui composa plusieurs petits ouvrages à contenu grammatical ${ }^{45}$. Le texte sur les composés est conservé dans (au moins) quatre manuscrits, dont le plus ancien est le manuscrit de Berlin Petermann 9 $(1260)^{46}$. Le manuscrit, fort endommagé, présente des lacunes importantes, qui peuvent être comblées grâce à la copie de la British Library, India Office 9, fol. 197 ${ }^{\mathrm{r}-\mathrm{v}}$, datant du XVII ${ }^{\mathrm{e}}-\mathrm{XVIII}{ }^{\mathrm{e}}$ siècle $^{47}$, qui semble contenir néanmoins quelques ajouts. La version contenue dans ce dernier manuscrit a été publiée, accompagnée d'une traduction anglaise, par Gottheil ${ }^{48}$. Nous proposons ici notre propre traduction, faite à partir du manuscrit :

La catégorie $(z n \bar{a})$ des noms composés. Une autre catégorie qui est appelée « composée », de deux noms complets (mšamlyē), comme Bar Hadbšabb $\bar{a}^{49}$ [fils du dimanche], Bar Šabtā [fils du šabbat], Benyamēn [fils de la droite], qui se traduit comme Bar Kä ${ }^{\prime} b y$ [fils de ma douleur] ${ }^{50}$, Yahb 'Alāhā [Dieu a donné], İšù yahb [Jésus a donné], Māran 'ammeh [le

42. Ou, selon Moberg, Jacques d'Édesse que Jean ne ferait que résumer.

43. Les éléments qu'il classe comme verbes ne sont en effet que des participes, cf. infra $\S 4.3$.

44. Traduction de l'allemand de Moberg 1909, p. 27. Moberg rapproche l'exemple bar-hānūm (nom propre d'origine biblique, $2 \mathrm{R} 23,10)$ du composé bar-dādeh de la Téchnè (cf. supra $\S 3.1$ ).

45. Sur cet auteur, voir BROCK 2011. Pour une liste des ouvrages, des manuscrits et de leurs éditions, voir FARINA 2018a, p. $245-246$.

46. Fol. $180^{\mathrm{r}}$. Le manuscrit a été copié dans la région de Bagdad, cf. SACHAU 1899, vol. 1, p. 321 et suivantes.

47. Furlani 1923. Une copie datée 1590 était conservée à Mossoul (cf. SCHER 1907, n. 111), mais jusqu'à présent nous n'avons pas pu la retrouver.

48. GotTHeIL 1893, p. 113-115.

49. Bar « fils de » + « un-dans-Šabbat » $>$ « fils du premier jour après le Šabbat » > « fils du Dimanche ».

50. Dans Gn 35,18 il est dit que Rachel, avant de mourir en couches, appela son dernier fils Ben 'Ônī « fils de ma douleur », mais qu'ensuite Jacob l'appela Benyamīn. Dans la
Gottheil $1887 ?$ ou pagination en romain? 
Seigneur est avec lui], 'Abdīšu $\bar{u}^{`}$ [serviteur de Jésus], Klīlīšu [couronne de Jésus], $\bar{I}_{s} \bar{u}^{c} d \bar{a} d^{51}$ [créé par Jésus] et tous ceux de cette catégorie. Autre catégorie. Une autre catégorie qui est appelée composée de trois noms complets, tels Tìmī Bar Tìmiñ $\bar{I}_{s} \bar{u}^{`}$ Bar Nūn et tous ceux de cette catégorie. Autre catégorie. Une autre catégorie est appelée composée de deux noms incomplets, tels krīshūd, [formé de] krstyānnā et īhūdā, protozmīa qui se traduit comme « limitation du temps » ${ }^{52}$, Tübalqayn [Tubal-Caïn Gn 4,22], qui est destructeur de Caïn, Sāmarā [Samarra] qui est en arabe sūrra man ra'a (celui qui voit se réjouit), c'est-à-dire, en arabe, sirūr [joie], Urišlem [Jérusalem], qui est nūra [lumière] et šlāmā [paix] et tous ceux qui sont de cette catégorie. Autre catégorie. Une autre catégorie est celle des composés [formés] d'une partie incomplète et d'une [autre] complète, tels Pnü'èl [Peniël, Gn 32,31], qui est le visage de Dieu, Šmū'èl, mlek malkē, rab baytā, qui est « le chef de la maison ».

Ce texte nous frappe à plusieurs égards. On observe d'abord une forte innovation dans le choix des exemples, dans la plupart des cas des noms d'individus (surtout des noms propres), issus des traditions onomastiques syrochrétienne et vétérotestamentaire. Deux toponymes sont aussi présents : Samarra et Jérusalem. Le premier situe clairement le traité de David dans un contexte araboislamique : l'étymologie proposée est celle qui remonte au calife al-Mu'tașim (794-842) ${ }^{53}$.

En outre, les diverses catégories de composés sont réinterprétées, par rapport à ce que nous avons observé chez Jean le Stylite, dans un sens qui nous paraît orienté vers la lexicographie. Dans une même classe, des composés constitués par deux noms « complets » se trouvent réunis avec des noms à la structure morphologique différente, tels que Bar Šabtā (composé de type a/) $\bar{I}^{5} \bar{u}^{c} y a h b$ (phrase nominale constituée par le nom propre $\bar{I} \bar{s}^{c} \bar{u}^{c}$ et par la troisième personne masculin singulier de l'accompli du verbe « donner »), Māran'ammeh (phrase nominale constituée par le nom Mār «Seigneur», le suffixe de

version syriaque Pešițtā Ben 'Ônī est en effet traduit comme Bar Kā’by.

51. Ce nom est un composé emprunté du moyen-perse, qui connait plusieurs anthroponymes composés d'un théophore et du participe $d \bar{a} d$ « fait » (cf. JulLien, JulLien \& GignouX, 2009).

52. Payne Smith 1879, vol. 2, p. 3252 : voir prōtesmīa du grec $\pi \rho 0 \theta \varepsilon \sigma \mu i \alpha \ll$ temps, moment établi ».

53. $E I^{2}$ vol. 8 , p. 1039 . Le toponyme connaît en réalité une tradition akkadienne, araméenne et persane. La fondation de la capitale abbasside date de 836 , ce qui fournirait aussi un ancrage chronologique ultérieur pour la biographie de David Bar Paulos. Il faut toutefois observer que le passage en question ne se trouve pas dans Petermann 9 et pourrait donc constituer un ajout tardif, ou du moins son attribution à David Bar Paulos n'est pas certaine. première personne pluriel -an, la préposition 'am et le pronom suffixe de troisième personne masculin singulier). Il nous semble qu'ici « complet » n'a pas le sens prégnant de nom à l'état emphatique, qu'il a acquis chez Jean le Stylite (et probablement chez Jacques d'Édesse). Au contraire, il paraît plutôt indiquer des noms qui, dans leur forme, font partie du lexique syriaque, ce sont des mots à part entière. D'ailleurs, même dans la Téchnè syriaque, le mot Bar dadeh (ou Bar Hadad) constitue le seul exemple de la dernière catégorie des composés, ceux dont le premier membre est " complet » et le deuxième " incomplet » (cf. 3.1). Il paraît donc que bar connaît une tradition interprétative comme forme lexicale indépendante. Cette interprétation nous semble d'autant plus nécessaire que la dernière catégorie évoquée par David inclut des exemples tels que mlek malkē, rab baytā, des composés formés par une construction génitivale dans lesquels on voit difficilement une différence morphosyntaxique par rapport à Bar Šabtā. D'ailleurs cette dernière classe se retrouve chez tous les grammairiens syriaques (voir supra et infra), toujours associée à l'exemple mlek malkē, et son interprétation nous semble bien établie.

La deuxième catégorie, en reprenant le modèle de la Téchnè, est celle des composés dont les deux membres sont incomplets, dans le sens qu'ils ne constituent que des morceaux de mots. David introduit des nouveautés importantes dans les exemples de cette classe, qui sont tous d'origine alloglotte (grecque, hébraïque, arabe). Le composé krīshūd, en particulier, a une structure insolite et hétérogène : la première partie est d'origine grecque, la deuxième sémitique. Le ms. Petermann 9 présente la

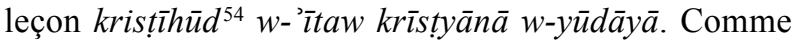
nous le verrons, cette deuxième forme se trouve également parmi les composés dans la «Grande grammaire » de Barhebraeus (m. 1286), le Ktābā d-șemhẹe.

\section{3/ La synthèse de Jean Bar Zo'bi}

La grammaire du syriaque oriental par Jean Bar Zocbi (XII ${ }^{\mathrm{e}}$-XIII ${ }^{\mathrm{e}}$ siècle) offre le traitement le plus long des noms composés, en résumant et réorganisant, au sein du chapitre consacré au nom, les descriptions des auteurs syriaques précédents ${ }^{55}$.

54. BäTHGEN 1880, p. 4* (trad. et commentaire p. 5, n. 1) intègre la même leçon dans une lacune du texte de la grammaire syriaque d'Élie de Tirhāan (m. 1049), dont Petermann 9 est le seul témoin.

55. Le texte de la grammaire de Bar Zo 'bi étant inédit, nous donnons une traduction sur la base du manuscrit British Library Add. 25876 , fol. $54^{\mathrm{v}}-56^{\mathrm{r}}$. Sur sa grammaire voir les nombreux travaux de Georges Bohas : BoHAs 2003a et b, 2005 et 2015-2016. 
Les schèmes ('eskīmē) du nom sont deux : simple (pšit țâ) et composé $(m r a k b \bar{a})$. Les autres grammairiens, je veux dire Mar 'Ahūhā d-emmeh, Yuhannān Esțūnāyā et Yuseph Hūzāyā, en suivant le modèle de la langue grecque, disent que les schèmes sont trois et ils introduisent comme exemple le nom substantif (šm ā qnōmāyā) 'Abrāhām.

En effet ils disent que les schèmes sont trois : simple ( $p$ šịt $t \bar{a})$ et composé $(m r a k b \bar{a})$ et plus que composé (yatīr men $m r a k b \bar{a}$ ). Le simple est comme 'ab, le composé est 'Abrām et le plus que composé est 'Abrāhām. En effet, dans sa forme simple [le nom] exprime la paternité qu'il [sc. Abram] aura. Ensuite, dans sa forme composée, il indique la paternité du peuple, qu'il détiendra à travers la naissance d'Isḥāq. Enfin, dans sa forme plus que composée, il indique la paternité sur les peuples qu'il détiendra à travers la naissance du Messie. En outre, dans sa forme simple, [le nom] proclame Dieu père, dans sa forme composée il exprime la substance du Fils et du Saint-Esprit; enfin, dans sa forme plus que composée il indique la foi et le baptême qui fut donné aux peuples par la venue du Messie. En effet, [les lettres] 'älap, bēt et rě̌ indiquent le Père et le Fils et le Saint-Esprit ${ }^{56}$. Ensuite, le he et le $\boldsymbol{m} \bar{\imath} m$ indiquent la foi et le baptême ${ }^{57}$.

De plus, dans sa forme simple, [le nom] indique le peu de connaissance qu'avaient de Dieu les générations d'avant Abrāhām. En effet, ils considéraient Dieu comme un homme. Dans sa forme composée il [le nom] indique la quantité moyenne de la connaissance qu'avaient de Dieu les générations qui viennent après la maison d'Abrāhām, jusqu'au lever du « Soleil de justice » ${ }^{58}$; même si elles considéraient Dieu comme pur esprit, elles le concevaient néanmoins comme limité. Dans sa forme plus que composée, [le nom] montre le plus haut degré de connaissance que le monde eut de Dieu à la venue du Messie.

Cette longue explication, au goût par endroits kabbalistique, tout en s'appuyant sur une citation littérale de la Téchnè d'Huzāyā, replace la triade 'ab-'Abrām'Abrāhām au sein de l'exégèse biblique, en la soustrayant à l'analyse grammaticale au sens propre ${ }^{59}$. Bar Zo'bi paraît partager l'embarras du lecteur moderne face à une analyse de la composition qui dépasse les limites de l'analyse grammaticale, tout en nous donnant une clé pour reconstituer son milieu d'origine visiblement exégétique.

Le grammairien peut ensuite aborder l'aspect plus proprement linguistique de la composition nominale, qu'il traite avec autant de détail :

56. En syriaque les premières lettres des mots ' $\bar{a} b$ « père », $b r \bar{a}$ « fils » et rū hă « esprit».

57. En syriaque hāymānnut $\bar{a}$ et $m a^{\circ} m \bar{u} d \bar{\imath} t \bar{a}$.

58. L'expression désigne le Christ.

59. Nous avons déjà vu (§ 4.1) que Jean le Stylite avait placé 'Abrām-'Abrāhām dans la section sur les schèmes du nom, en les séparant du traitement détaillé des composés.
Le saint Mār Élie de Șōbā ${ }^{60}$ et d'autres avec lui divisent le schème [du nom] en deux catégories, selon ce qui est plus approprié pour la langue syriaque : simple et composé. Simple ce sont [des noms] comme 'Elìyā, Bașlī'êl, Yōhannān et tous les noms d'individus (qnōmāyēe). Et il faut savoir que les noms d'individus sont simples quant à [leur] son, car chacun d'entre eux désigne un individu $(q n \bar{o} m \bar{a})$. Quant à [leur] signification (sūkālā), ils sont pour la plupart composés, comme Yōhannān [évoqué] ci-dessus et 'Abrāhām.

Le deuxième schème est composé quant à [leur] son, tandis qu'il est simple quant à [leur] signification, comme celui qui indique un individu (had qnōmā). Sa composition se fait de plusieurs manières. Certains [noms] sont composés de deux noms complets (mšamlyē), tel Yāhū 'āhăz. D'autres [sont composés] d'un nom complet et d'un nom incomplet (lā mšamlyā), comme mlek malke $\bar{e}^{61}$, pìl tayšā, 'nez 'aylā. D'autres [sont composés] d'un adjectif prédicatif (melat $\check{s} m \bar{a})$ et d'un nom incomplet, comme 'ahì d-kul, qaddīš-kul. D'autres sont composés d'un verbe et d'un nom complet, comme šātē hamrā et pālah 'ar'áa. D'autres [sont composés] de plusieurs membres, comme quand je dis $l \bar{a}$ y $\bar{a} d a^{c}$ napšeh [qui ne se connaît pas soi-même], lā 'dīl b-dūbreh [irréprochable dans sa conduite], et comme šārē hayklāa u-bārē leh la-tlātā yawmīn [lit. celui qui détruit le temple et qui le bâtit en trois jours]. Et ceci aussi est un schème composé, qui indique un nom substantif ${ }^{62}$.

Bar Zo`bi semble opérer une première distinction entre les composés qui sont des noms d'individus (ou ici noms propres) et le reste des composés ${ }^{63}$. Les noms

60. Grammairien syriaque, mort en 1046, auteur d'une grammaire ainsi que d'un vocabulaire syriaque-arabe. Selon Bar Zo 'bi, Mār Elie de Șōbā serait le premier à avoir renoncé à la catégorie du plus que composé. Comme l'observait Gottheil (GotTHEIL 1887,p. 10 n. a), ce passage ne se trouve pas dans la grammaire d'Élie que nous possédons (le Tūrāṣ mamllā).

61. Le manuscrit de Londres lit malkā, mais d'autres témoins, comme le manuscrit de la cathédrale chaldéenne de Mardin CCM 20, fol. 24r, lisent malkē.

62. Bar Zo'bi discute ensuite des cinq espèces du discours ( $p \bar{a} q \bar{o} d \bar{a}$ « impératif », mša lānā « interrogatif », metkašpāna

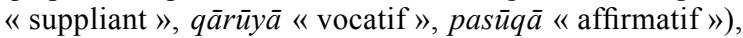
tout en intégrant une bonne partie des composés dans ses exemples. Il en profite ainsi pour renseigner le lecteur sur le fait que le pĭl tayš $\bar{a}$ (éléphant-chèvre) n'existe pas, tandis que mlek malkē (roi des rois) et 'ahīd-kul (tout-puissant) signifient bien quelque chose (cf. BL Add 25876 , fol. $56^{\mathrm{V}}-57^{\mathrm{r}}$ ).

63. La catégorie la plus générale des noms selon Bar Zo`bi est celle des substantifs (ou « naturels », syr. kyānāyā), qu'il définit comme « tous ceux qui indiquent la substance (ou "nature" kyānā) des choses qui sont dans cet univers" (BL Add 25 876, fol. 38 r voir aussi BoHas 2015-2016, p. 28). Les noms d'individus (qnōmāyē) sont subordonnés aux noms substantifs parce que la substance se divise en plusieurs espèces, alors que l'individu ne le peut pas ( « les noms d'individus sont tous ceux qui se distinguent l'un de 
d'individus constituent une unité phonologique, alors que leur sémantique est composée : ainsi 'Elìya a (hébr. 'Elìyahô) signifie " $\mathrm{Ya}(\mathrm{hu})$ est mon dieu ${ }^{64}$. À cette catégorie s'oppose celle des noms qui sont « composés quant au son », mais simples quant à la signification. Cette opposition apparemment difficile à saisir devient claire si l'on songe aux exemples et, en particulier, si l'on oppose les cas de 'Abrāhām et de 'nez 'aylā. 'Abrāhām est analysé comme état construit de type a/ ( ${ }^{\prime} A b+r a \bar{a} h \bar{a} m$ « père des multitudes ») ayant subi un processus d'univerbation. Il désigne un individu, mais son sens est analysable en tant que composé : d'après sa reconstruction, Bar Zo bi peut ainsi affirmer que Abraham est le père des multitudes. Au contraire 'nez 'aylā " gazelle » (voir supra § 2) est un composé de type a/ dont les membres restent distincts, mais dont le sens n'est pas analysable : une " gazelle» n'est pas la chèvre d'un cerf, ni un mélange de chèvre et de cerf. Avec les seuls ajouts du nom propre Yāh $\bar{u}$ 'a $h \bar{a} \bar{z} z$ et des composés " démolisseur et bâtisseur du temple » (paraphrase de Jn 2,19), cette deuxième section recoupe les mêmes exemples que nous avons trouvés chez Jean le Stylite $(\S 4.1)$. Toutefois, Bar Zo'bi introduit une distinction ultérieure au sein des composés d'un verbe et d'un nom, en distinguant ceux qu'il qualifie d'adjectifs ${ }^{65}$, comme 'ahì d-kul « tout-saint», de ceux qu'il qualifie de verbes, comme šătē hamrā «buveur de vin ». Ces deux classes de composés étaient distinguées chez Jean le Stylite par rapport à la morphologie du deuxième élément, selon qu'il est « complet » (c'est-à-dire à l'état emphatique, comme hamrā) ou incomplet (comme kul). Bar Zo'bi, quant à lui, oriente son classement par rapport au premier élément. Quelle est donc la différence entre 'ahìd (participe passif à l'état construit du verbe 'ahad « tenir ») et šătē (participe actif à l'état construit du verbe štā « boire ») ? La forme 'ahīd est considérée par Bar Zo bi comme un adjectif qualificatif (geď̄ānāy $\bar{a}$ « d'accident », cf. BL Add 25876, fol. $127^{\vee}$ ), tout comme les formes qaddī̌s « saint », ou zaddīq « juste», formées selon le même paradigme morphologique. Par contre, il considère

l'autre par les espèces de la substance. En effet, l'individu se distingue de la substance en cela que la substance se divise en plusieurs espèces [...] l'individu n'en est pas capable. » BLAdd 25876 , fol. $42^{\mathrm{r}}$., voir aussi BoHAs 2015-2016). Cette hiérarchie correspond précisément, jusque dans les termes de sa formulation, à celle que Bar Zo'bi établit dans son traité théologique (trad. et commentaire dans FURLANI 1926), cf. sur le même sujet FARINA à paraître.

64. Il est intéressant d'observer que les noms de cette première classe sont tous des noms d'origine hébraïque.

65. La définition que Bar Zo 'bi donne du melat šm $\bar{a}$ « verbe du nom » est : toutes les voix dont on dit qu' « elles remplissent la place du nom et du verbe » (BL Add 25876, fol. 127' ). Pour cette interprétation de melat šm $\bar{a}$, voir aussi MoBERG 1907-1913, vol. 2, p. 56*. šătē comme une forme de nom d'agent (participe), et donc une forme verbale.

\section{4/Unenouvelle systématisation: Grégoire Barhebraeus}

Le polymathe Grégoire Barhebraeus, mort en 1286, auteur de deux traités grammaticaux, est le dernier représentant de la tradition grammaticale syriaque avant le $\mathrm{XVI}^{\mathrm{e}}$ siècle $^{66}$. Ses œuvres sont considérées comme une synthèse et une harmonisation de la tradition grammaticale gréco-syriaque et de la linguistique arabe, qu'il utilise comme source complémentaire dans sa première Grammaire métrique ${ }^{67}$ et comme modèle doué d'une sens? majeure capacité structurante dans le Ktāba $\bar{d}$-semhēe (« le livre des éclairages »), sa garnde grammaire de la langue syriaque $^{68}$.

La Grammaire métrique est une grammaire en vers, que Barhebraeus a complétée par un apparat de gloses et d'explications, qui court tout au long du poème. Les composés y sont traités dans le premier des quatre chapitres de l'œuvre, consacré au nom ${ }^{69}$, et en particulier dans la section sur les schèmes ${ }^{70}$ :

Les schèmes sont comme suit

comprends-moi, ô jeune perspicace :

simple, comme $Y \bar{a} h \bar{u}$ ' $\bar{h} h \bar{a} z$

et composé, $Y \bar{a} h \bar{u}^{2} \bar{a} h \bar{a} z$.

Deux gloses sont ajoutées par Barhebraeus, qui complètent cette formulation très synthétique :

[A.] [simple] C'est-à-dire, lorsque tu dis juste $Y \bar{a} h \bar{u}$ à quelqu'un, ou à d'autres.

[B.] [composé] C'est-à-dire, lorsque tu appelles un individu

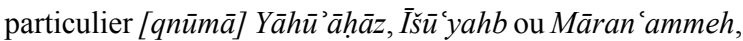
ou tout autre nom composé.

[C.] Il faut savoir que les composés sont constitués soit de deux noms complets (mšamlyē), comme Zōrbābēl et $Y \bar{a} h \bar{u} \bar{a} h \bar{a} z$; soit de deux noms incomplets (hasìrê), comme kleb bar'nāšā [le chien de l'homme] ou patryark $\bar{a}$ [patriarche], où, par rapport à *patrirkōnītā, quatre lettres ont été éliminées; soit de trois noms incomplets, comme $\bar{I} s \bar{u}$ bar Nūn [Jésus fils de Nun] ou Beldān bar Beldān [Beldan fils de Beldan]; soit d'un nom et d'un verbe, comme pālah

66. Pour des renseignements biographiques, voir TAKAHASHI 2011.

67. Texte syriaque éditée par MARTIN 1872.

68. Edition Moberg 1922, traduction allemande Moberg 19071913.

69. Les autres chapitres sont consacrés au verbe, à la conjonction et à la syntaxe. Selon le modèle arabe (mais aussi dans la lignée de la tradition du Peri hermeneias), Barhebraeus reconnait trois parties du discours : le nom, le verbe et la conjonction. Sur la combinaison de modèles grecs et arabes dans la Grammaire métrique voir FARINA 2015 et 2017.

70. MARTIN 1872, p. 9-10. 
'ar' $\bar{a}$ ou rāḥem kespā [qui aime l'argent], ou qāṭel 'nāšse [tueur d'hommes, homicide]; soit d'un nom incomplet et d'un nom complet, comme pĭl tayšā ou sūsay tawrā [taureaucheval].

Par rapport à la tradition précédente, nous pouvons retrouver des éléments de continuité dans le choix des exemples. Yāhù $\bar{a} h \bar{a} z$ révèle une dépendance de Bar Zo 'bi, qui semble avoir été le premier à l'introduire dans le traitement des composés ${ }^{71}$. Par ailleurs, Barhebraeus y apporte aussi une certaine variation, enrichissant le corpus des exemples par rapport à la tradition.

Patryarkā représente le tout premier vrai composé grec, emprunté par le syriaque, qui soit utilisé pour illustrer la structure de la composition nominale. Quant à sa décomposition étymologique, la tradition manuscrite présente plusieurs variantes : le ms. Or. 298, copié en 1360 , présente au fol. $7^{\mathrm{r}}$ la forme patryrkōnītā [sic!], alors que d'autres manuscrits plus tardifs mais de tradition indépendante, comme le ms. CFMM 500 de l'église des Quarante-Martyrs de Mardin, fol. $8^{\mathrm{r}}$ (copié en 1480) ${ }^{72}$, offrent la leçon plus complexe patīr wa-'rkōnițā. Dans ce dernier cas les deux membres du composé sont bien distincts, même si Barhebraeus considère *-arkā comme une forme abrégée du mot 'arkōnitta , autre emprunt grec bien documenté en syriaque au sens de " prince, chef $\rangle^{73}$.

Ce qui frappe dans cette glose est une totale inversion de l'interprétation des noms comme $\bar{I} s \bar{u} \bar{u}^{c}$ bar Nūn, par rapport à ce que nous avons vu chez David bar Paulos, puisqu'ils sont ici considérés comme étant composés de trois membres « incomplets ». Il n'est donc plus question de considérer bar comme un mot « complet » ni comme une forme lexicalisée.

$\mathrm{Au}$ cours de la tradition manuscrite, d'autres gloses ont été ajoutées par les copistes, parmi lesquels le plus important est Daniel de Mardin, auteur de la plus ancienne copie datée de cet ouvrage (BML Or. 298, 1360). La particularité des gloses de Daniel est qu'elles sont rédigées en grande partie en arabe et touchent surtout à une comparaison entre syriaque et arabe. En outre, lorsqu'il rencontre un terme syriaque d'origine grecque, il essaye d'en reconstruire la forme originaire et de la transcrire en caractères grecs ${ }^{74}$. Dans le passage en question, Daniel semble avoir été intrigué par l'étymologie pațyarkā de patryrkōnițā et il propose dans la marge l'étymologie

compléter

de ou que ?
71. Une dépendance commune d'Élie de Nisibe, que Bar Zo bi cite comme source, est aussi envisageable, mais nous ne possédons pas le texte d'Élie (cf. supra n. **).

72. On retrouve cette leçon, par exemple, dans le manuscrit d'Alqoš DCA 505, fol. 11v (copié par 'Abdišo ' de Gazarta en 1552), issu de la même tradition de CFMM 500, ainsi que dans BnF syr. 261, fol. $4^{\mathrm{r}}$, dont la position reste à déterminer. 73. Payne Smith 1879, vol. 1, p. 385.

74. Sur les gloses gréco-arabes de Daniel, voir Farina 2017. grecque correcte, accompagnée par une traduction littérale en (moyen-arabe) des deux membres du composé : grec $\pi \alpha \tau \rho \iota \alpha ́ \rho \chi \eta \varsigma$, ar. rayyis 'al-'abā (chef du père) $)^{75}$.

Dans le Ktābā $d$-șemḥe, Barhebraeus, en suivant en quelque sorte l'organisation théorique de Bar Zo'bi, sépare le traitement des schèmes du nom de celui, plus spécifique, des noms propres. D'abord, dans le chapitre sur le nom, dans la section sur le nom d'individu (qnūmāyā), il donne les précisons suivantes ${ }^{76}$ :

Le nom d'individu peut être simple, comme 'ab, composé, comme 'Abrām, ou plus que composé, comme 'Abrāhām, dérivé, comme $Y a^{`} q \bar{u} b$ de 'eqbā [pied, cheville], ou temporaire, comme šemša [soleil], mot par lequel les gens sont parfois appelés sans qu'il y ait une vraie similitude.

Le nom d'individu composé est constitué soit par deux noms complets (šalmē), comme Yāhū'āhăaz; soit par deux noms incomplets (hasīrēe), comme Melkīzedeq; ou encore par un nom incomplet et un nom complet, comme rabbaytā; ou par un verbe et deux noms : un agent caché (gnizza) et un patient pronominal (hūšs $\bar{a} y \bar{a})$, comme dans Nasrā $k$ dans le livre des Rois [2 R 19,37] : $\bar{u}$-kad sged hwā bēt Nasrāk 'alāhāhe ${ }^{77}$; ou par une particule et un verbe comme $l \bar{a}$ 'etrahmat [non aimée, Os 2,25]; ou par trois noms, comme $\bar{I} \bar{s}^{\prime} \bar{u}^{c}$-bar-Nūn; ou deux mots joints (trēn malwātāa $)^{78}$, comme 'Ahūh-d-'emmeh, 'Abd-Ǐš $\bar{u}^{c}$ [serviteur de Jésus]; ou encore il s'agit d'une phrase [composée] d'un nom et d'une particule, comme 'Immanu' 'él [Dieu est avec nous] ou d'un nom et d'une forme verbale à l'indicatif, comme Slì $\bar{a} \bar{a}-z k \bar{a}$ [la croix a vaincu], Yahb-'allāhā [Dieu a donné], ou par un nom et un verbe à l'impératif, comme Sūud 'aylē [chasse-cerfs] ${ }^{79}$, ou par deux verbes comme msarheb $s ̌ b \bar{a}$ [qui se hâte de ravager], tkeb $b \bar{a}^{3} e z$ [qui exige de saccager, Is 8,3].

Le paragraphe s'ouvre avec des noms d'individus, issus de la tradition vétérotestamentaire, pour se poursuivre avec des incursions dans l'onomastique syriaque postérieure.

Dans son analyse de la syntaxe des composés, il puise aussi dans la théorie linguistique arabe, en introduisant de nouvelles catégories. D'après Moberg ${ }^{80}$, les termes syriaques $h \bar{u} \bar{s} b \bar{a} y \bar{a}$ lit. « sous-entendu » et gnīzā « caché »

75. Ms. BML Or. 298 fol. $7^{\text {r }}$

76. Texte syriaque dans Moberg 1922, p. 7-8, traduction allemande MOBERG 1907-1913, p. 14-15.

77. « Comme il était prosterné dans la maison de Nasrāk son dieu $»$.

78. Sc. par deux membres d'une annexion.

79. Ce nom propre nous est connu par Stephanos Bar Sūd 'aylē, mentionné comme hérétique dans le Chronicon ecclesiasticum de Barhebraeus 221.14 (cf. PAYNe SMITH 1879 , vol. 2, p. 3377). Barhebraeus interprète Sūd comme un impératif du verbe $s \bar{a} d$ « chasser », d'une racine de moyenne faible $\sqrt{s} w / y d$.

80. Moberg 1907-1913, vol. 2, p. 25*, 42*. 
traduisent respectivement les notions arabes de damīr « pronom » et de (damīr) mustatir «(pronom) caché ». $H \bar{u} \bar{s} b \bar{a} y \bar{a}$ indique chez Barhebraeus « seulement le pronom personnel et le démonstratif $»^{81}$, donc dans le cas du nom Nasrā $k$, le pronom objectif suffixe $-\bar{a} k\left(2^{\mathrm{e}}\right.$ personne masc. sg.). Le concept de (damīr) mustatir « (pronom) caché » correspond au sujet pronominal implicite dans une forme verbale donnée : dans le verbe arabe kataba « il a écrit », on peut reconstruire un sujet pronominal huwa « il », qui est « caché ». Ainsi, si je dis kataba Zaydun «Zaid a écrit», l'agent $\left(f f^{`} i l\right)$ de la phrase est explicite et identifiable à Zaid. Si, par contre, je me limite à dire kataba « il a écrit », l'agent est implicite et s'identifie avec le « pronom caché » huwa « il».

Selon l'analyse de Barhebraeus, le nom du dieu Nasrā $k^{82}$ serait donc composé de la troisième personne de l'accompli masculin singulier du verbe $n s a r$ « il a scié », du pronom agent « caché » $h \bar{u}$ « il » et du pronom suffixe de deuxième personne masculin singulier $-\bar{a} k$ qui exprime le patient : « il t'a scié ».

Dans le quatrième chapitre du livre consacré au nom, après avoir donné une définition du schème comme « une figure vocale qui est transmise à l'oreille par la prononciation d'une composition de lettres ${ }^{83}$, Barhebraeus traite des schèmes des noms communs (gensānāyā) :

Il en est de la composition des noms communs comme de celle des noms d'individus, la composition commune étant soit manifeste (gâlēe), comme pīl tayšă, 'nez 'aylā, gāmal nemrā [chameau-léopard ${ }^{84}$ ], sūsay barnāš [cheval-homme $>$ centaure], gbarnāšāa [homme-femmme $>$ hermaphrodite], krisțīhūd [judéo-chrétien] ${ }^{85}$; soit opaque (kāsēe), comme 'amzartā [pygmée] chez Mār Ephrem : « les géants que j'avais générés étaient devant lui comme des pygmées », c'est-à-dire comme 'amtā [coudée] et zartā [empan], et ainsi les signifiés des parties sont clairs, tandis que le sens du composé est opaque. Et c'est le contraire dans le cas du nom $b^{`} e l d b a \bar{a} b \bar{a}$ [ennemi, diable], pour lequel le sens du composé est clair, mais celui des parties est opaque.

Dans cette section, Barhebraeus s'intéresse à la forme des composés, en distinguant entre ceux dont les deux éléments sont bien distincts, comme 'nez 'aylā, et ceux dont les deux éléments source ne sont pas faciles

81. Moberg 1907-1913, vol. 2, p. 42*.

82. La forme de ce nom que l'on trouve dans la Bible hébraïque, Nisrok, est en effet associée à un des noms sémitiques de l'aigle : ar. nisr, heb. néšer, syr. nešrā, cf. GeSENIUS 1846, p. 554.

83. Moberg 1922 , p. 22, traduction allemande Moberg 19071913, vol. 1, p. 45.

84. Cf. lat. camelopardus, http://liber-glossarum.huma-num.fr/ search.html?id=\& form $=$ camelopardus $\&$ def $=$

85. Cf. supra $\S 4.2$. à identifier, comme 'amzartā "pygmée », qui serait composé de 'amtā « coudée » et zartā « empan » (des gens qui ont la coudée de la longueur d'un empan?). Dans ce dernier cas, Barhebraeus observe que le sens du composé ne dérive pas de la compréhension des signifiés des deux parties. Inversement, le composé $b^{`} e l d b \bar{a} b \bar{a}$ a un sens bien connu d' « ennemi, adversaire » qui est, par excellence, le « diable », mais sa structure n'est pas transparente. Le mot dérive de l'univerbation du syntagme $b^{`} e l d b a \bar{b} b \bar{a}$, dérivé, selon Sokoloff ${ }^{86}$, de l'akkadien bel dabābi « ennemi, adversaire dans un litige $»^{87}$.

\section{5/ CONCLuSIONS}

Plusieurs réflexions peuvent émerger de cette étude. Nous nous limiterons ici à quelques observations à caractère général.

D'un côté, la persistance du modèle de la Téchnè syriaque semble demeurer à travers les siècles, pour l'organisation de la matière grammaticale. L'importance fondamentale de la triade 'ab, 'Abrām, 'Abrāhām en fait l'exemplum du composé chez la plupart des auteurs et donne une justification théologique à la composition des mots, en tant que processus de création.

En outre, l'association privilégiée de la composition nominale avec les noms propres, qui gardent toujours une place prééminente dans l'illustration de cette catégorie, nous paraît aussi un héritage du premier modèle grec de la Téchnè. Il est bien vrai que l'anthroponymie est un domaine privilégié de la composition, mais la morphosyntaxe des noms propres composés ne diffère aucunement de celle des noms communs et ne suffit pas, à notre avis, à en expliquer l'émergence. Au fil des siècles, d'autres classes (morpho)sémantiques s'établissent : les noms d'animaux, les attributs divins, les composés participiaux.

Mais c'est surtout le classement des composés en raison de la forme " complète » (mšamlya $)$ ou "incomplète » (hasìr $\bar{a}$ ) de leurs membres qui reste le critère analytique principal de toutes les descriptions que nous venons de parcourir.

De l'autre côté, le contenu métalinguistique attribué aux termes mšamlyā et hasīrā et le sens de ce classement varient d'un auteur à l'autre et à travers les époques. Dans la Téchnè syriaque les deux termes ont un sens générique, dépourvu d'une valeur morphologique précise. L'organisation des exemples chez Jean le Stylite révèle, inversement, le développement d'un signifié métalinguistique plus précis : les noms mšamlyē sont ceux qui possèdent la terminaison de l'état emphatique

86. SoKoloff 2002, vol. 1, p. 227 ; voir aussi SokOLOFF 2009, p. 171 ; PAYNe SMith 1879, vol. 1, p. 562.

87. Littéralement « le maître du discours ». 
$(-\bar{a},-\bar{e}$, etc. $)$, tandis que les hasìrēe sont ceux qui en sont dépourvus (qu'ils soient à l'état construit ou absolu). C'est une caractéristique générale de la langue syriaque (l'interaction de plusieurs formes du nom) qui émerge à travers l'analyse des mécanismes de la composition nominale. Cette valeur métalinguistique prégnante ne semble pas présente chez David Bar Paulos, qui a une conception apparemment plus proche de la Téchnè, ou, de toute façon, plus orientée vers l'intérêt lexicographique des composés. Par contre, elle est retenue par Bar Zo bi et Barhebraeus, tout en se croisant avec d'autres catégories plus complexes, comme celles de l'adjectif prédicatif (melat šmāa $\S 4.3$ ) ou de l'annexion (trēn malwātāa, Kt. $d$-S.. § 4.4), dont elle constitue la prémisse théorique.

Les chaînes de transmission des exemples nous paraissent également intéressantes. De la Téchnè syriaque, seuls 'Abrāhām et mlek malkē survivent tout au long de la tradition. Par contre, un exemple maladroit comme Müšse n'a pas connu de suite, même si la catégorie des composés troubles, dont les deux membres sont « incomplets", revient à maintes reprises. Il est d'ailleurs significatif que les exemples de cette classe soient très instables et varient d'un auteur à l'autre.

Le lien entre la tradition logique et celle de la grammaire se fait par l'intégration de 'nez 'aylā et de 'Ahūh-d-'emmeh au corpus des exemples de Jean le Stylite, Bar Zo'bi et Barhebraeus.

Le groupe pālah 'ar'ā, šātē hamrā, pīl tayšă, 'nez 'aylā, mlek malkē, qaddī̌s-kul, 'ahìd-kul, lā yāda' napšeh, $l \bar{a}$ ' $d \bar{l} l b$-dūbreh, qui figure pour la première fois dans son ensemble chez Jean le Stylite (mais en remontant peut-être à Jacques d'Édesse) est entièrement repris par Bar Zo 'bi, tandis que Barhebraeus en fait une sélection partielle (avec une certaine variation entre ses deux grammaires), qui confirme toutefois une continuité dans la tradition grammaticale. Ce dernier présente d'ailleurs la plus grande variété d'exemples, en enrichissant chaque catégorie par des composés inédits.

À cet égard, le texte de David Bar Paulos paraît assez isolé, ne partageant que l'exemple mlek malkē avec la tradition qui le précède, et n'étant repris que par Barhebraeus, et seulement pour une petite portion de sa

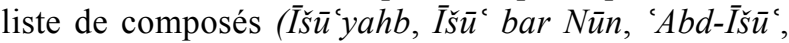
Yahb-'allāhā, rabbaytā, krisțīhūd).

Les matériaux présentés dans cet article ouvrent plusieurs autres questions et une étude approfondie de leurs contextes, à la fois linguistiques, historiques et littéraires, est nécessaire, afin d'en saisir toutes les implications. Nous considérons cet aperçu comme une invitation à puiser dans la richesse et la complexité de la pensée linguistique syriaque, et nous nous proposons d'approfondir certaines des pistes ouvertes par cette première présentation dans des publications à venir.

\section{CNRS, Histoire des théories linguistiques,}

email à renseigner

Université de Paris 7

\section{BIBLIOGRAPHIE}

BÄTHGEN F.

1880 Syrische Grammatik des Mar Elias von Tirhan, Leipzig.

BELARDI W.

1985 Filosofia, grammatica e retorica nel pensiero antico, Roma.

BoHas G.

2003a «Comment Bar Zu'bî se passe de la notion de racine », dans $* * * *$ (éd.), Comunicazione al Colloquio Le patrimoine syriaque, Damas, p. ${ }^{* * * * *}$.

$2003 b$ « Les accidents du verbe dans la grammaire de Bar Zu'bî ou : une adaptation de la Technê », Langues et littératures du monde arabe 4, p. 54-86.

2005 Les bgdkpt en syriaque selon Bar Zo'bî, Toulouse.

2015-2016 «Définition du substantif et catégorisation des choses qui sont dans l'univers chez Bar Zo 'bī », Mélanges de l'université Saint-Joseph 66, p. 21-40.

Briquel-Chatonnet F., Debié M.

2017 Le monde syriaque, Paris.

BROCK S.

1977 « Greek into Syriac and Syriac into Greek», Journal of the Syriac academy 3, p. 406-422.

1979 " Aspects of translation technique in antiquity », Greek, Roman and Byzantine studies 20, p. 69-87.

1991 «The Syriac background to Hunayn's translation techniques », Aram 3, p. 139-162.

2004 «Changing fashions in Syriac translation technique : the background to Syriac translations under the Abbasids ", Journal of the Canadian society for Syriac studies 4, p. 3-14.

2007 «Translation: Greek and Syriac », dans A history of ancient Greek : from the beginnings to late antiquity, ed. by A. F. Christidis, Cambridge - New York Melbourne, p. 935-946, 957-959.

2011 « Dawid bar Pawlos », GEDSH, p. 116-117.

ButTs A. M.

2011 « Hunayn b. Isḥāq », GEDSH, p. 205-206.

CAtagnoti A.

2005 «Traditions onomastiques sémitiques dans la documentation du III ${ }^{\mathrm{e}}$ millénaire », dans Proceedings of the $10^{\text {th }}$ meeting of Hamito-Semitic (Afroasiatic) linguistics, Florence, 18-20 April 2001, ed. by P. Fronzaroli \& P. Marrassini, Firenze, p. 145-154.

CianCAglini C.

2008 Iranian loanwords in Syriac, Wiesbaden. 
je propose de le supprimer (cf. n. 2)
2010 « Calchi e paretimologie dotte nell'interferenza siroiranica », Incontri linguistici 33, p. 187-205.

Ciancaglini C., Alfieri L.

2013 «Iranian and Greek influence on the Syriac lexicon: the emergence of compound words », Incontri linguistici 36, p. 109-138.

CONTINI R.

2001 «Storia della scienza siriaca. 3, Le scienze del linguaggio », dans Storia della scienza. 4, Medioevo, Rinascimento, Roma, p. 26-36, 68-69.

DACCACHE J.

DUVAL R.

1881 Traité de grammaire syriaque, Paris.

1901 Lexicon Syriacum auctore Hassano Bar Bahlule, Paris.

EI $I^{2} \quad$ Encyclopédie de l'Islam, nouvelle édition, Leiden Paris, 1954-2009.

FARINA M

2015 «La Grammatica metrica di Barhebraeus (XIII sec.) e le sue glosse : siriaco, greco e arabo in contatto", dans Rappresentazioni linguistiche dell'identità, a cura di M. BeNEDETTI (Quaderni di $A I \Omega N$ NS 3), Napoli 2015, p. 107-125.

2017 « The Syro-Arabic glosses to Barhebraeus' Metrical grammar ", dans Language and identity in multilingual Mediterranean settings: challenges for historical sociolinguistics, ed. by P. Molineldi, Berlin - Boston, p. 157-170.

2018a « Manuscrits de grammaires et lexiques syriaques », dans Les auteurs syriaques et leur langue, éd. par M. FARINA (Études syriaques 15), Paris, p. 243-254.

2018 b « La théorie linguistique de Jacques d'Édesse », dans Les auteurs syriaques et leur langue, éd. par M. FARINA (Études syriaques 15), Paris, p. 167-187.

à paraître «Bar Zobi's grammar and the texture of knowledge in $13^{\text {th }}$ century ", dans The impact of 'Abdīshō' bar Brīkhā: papers collected on his $700^{\text {th }}$ anniversary, ed. by Z. Paša \& S. Rassi, Rome.

FURLANI G.

1923 « Il manoscritto siriaco 9 dell'India Office », Rivista degli studi orientali 10 , p. 315-320.

1926 « Giovanni Bar Zô‘bî sulla differenza tra natura ed ipostasi e tra persona e faccia ", Studi e materiali di storia delle religioni 2, p. 230-242.

GEDSH Gorgias encyclopedic dictionary of the Syriacheritage (GEDSH), ed. by S. BRock et al., Piscataway NJ, 2011.

GensLer O. D.

2011 «Morphological typology of Semitic», dans The Semitic languages : an international handbook, ed. by S. WeNINGER et al., Berlin - Boston, p. 279-302.

Gesenius F. W.

1846 Hebrew and Chaldee lexicon of the Old Testament scriptures, London.
Gignoux Ph.

2011 Lexique des termes de la pharmacopée syriaque, Paris.

GotTheIL R. J. H.

1887 A treatise on Syriac grammar by Mâr(i) Eliâ of Sôbhâ, ed. and transl. from the manuscripts in the Berlin Royal Library by R. J. H. GotтheIL, Berlin.

1893 « Dawidh bar Paulos, a Syriac grammarian », Journal of the American Oriental society 15, p. CXI-CXVIII.

Gutas D.

1998 Greek thought, Arabic culture: the Graeco-Arabic translation movement in Baghdad and early 'Abbāsid society $\left(2^{\text {nd }}-4^{\text {th }} / 5^{\text {th }}-10^{\text {th }} c\right.$. $)$, London - New York.

HAdDAd B., IsAaq Y.

1988 Syriac and Arabic manuscripts in the Library of the Chaldean Monastry Baghdad. 1, Syriac manuscripts, Baghdad.

Hoffmann J. G. E.

1869 De Hermeneuticis apud Syros Aristoteleis, Leipzig.

HuEHNERGARD J.

2005 A grammar of Akkadian, Winona Lake IN.

HugONNARD-Roche H.

2004 La logique d'Aristote du grec au syriaque : études sur la trasmission des textes de l'Organon et leur interprétation philosophique, Paris.

2018 «La tradition du Peri hermeneias d'Aristote en syriaque, entre logique et grammaire ", dans Les auteurs syriaques et leur langue, éd. par M. FARINA (Études syriaques 15), Paris, p. 55-93.

Jullien C., Jullien F., Gignoux Ph.

2009 Iranisches Personennamenbuch. 7, Iranische Namen in semitischen Nebeüberlieferungen. 5, Noms propres syriaques d'origine iranienne, Wien.

LALLOT J.

1998 La grammaire de Denys le Thrace, trad. et annotée par J. LALlot, $2^{\mathrm{e}}$ éd. revue et augmentée.

LIVERANI M.

2003 Oltre la Bibbia, Bari.

MARTIN J.-P.-P.

1872 Euvres grammaticales d'Abou 'lfaradj dit Bar Hebreus, éd. par l'abbé MARTin, Paris.

Merx A.

1889 Historia artis grammaticae apud Syros, Leipzig.

Moberg A.

1907-1913 Buch der Strahlen : die grössere Grammatik des Barhebräus, Leipzig.

1909 « Die syrische Grammatik des Johannes Esț̄nājā », Le monde oriental 3, p. 24-33.

1922 Le livre des splendeurs : la grande grammaire de Grégoire Barhebraeus, texte syriaque éd. d'après les manuscrits avec une introd. et des notes par A. Moberg, Lund.

MONTANARI E.

1984-1988 La sezione linguistica del Peri hermeneias $d i$ Aristotele. 1-2, Firenze. 
MutZafi H.

2008 The Jewish Neo-Aramaic dialect of Betanure (province of Dihok), Wiesbaden.

NÖLDEKE T.

1898 Kurzgefasste syrische Grammatik, Darmstadt.

PAyne Smith R.

1879 Thesaurus Syriacus, Oxford.

Pollak I.

1913 Die Hermeneutik des Aristoteles : in der arabischen Übersetzung des Ishāk Ibn Honain, Leipzig.

PRITCHARD J. B.

1969 Ancient Near Eastern texts relating to the Old Testament (ANET), Princeton NJ, $3^{\text {rd }}$ ed. with supplement.

SACHAU E.

1899 Die Handschriftenverzeichnisse der königlichen Bibliothek zu Berlin, Berlin.

Schall A.

1960 Studien über griechischen Fremdwörter im Syrischen, Darmstadt.

SCHER A.

1906 « Notice sur les manuscrits syriaques conservés dans la bibliothèque du couvent de Notre-Dame-desSemences », Journal asiatique $10^{\circ}$ serie, 7, p. 479$512 ; 56-82$.

1907 « Notice sur les manuscrits syriaques conservés dans la bibliothèque du patriarcat chaldéen de Mossoul », Revue des bibliothèques 17, p. 227-260.

Sснмітт R.

2013 «Bisotun. 3, Darius's inscriptions», dans Encyclopedia Iranica. 4, 3, p. 299-305.

SoKolofF M.

2002 A dictionary of Jewish Babylonian Aramaic of the Talmudic and Geonic periods, Ramat-Gan Baltimore.

2009 A Syriac lexicon: a translation from the Latin, correction, expansion, and update of C. Brokelmann's Lexicon Syriacum, Winona Lake IN - Piscataway NJ.

Strohmaier G.

2010 «Ishāạk b. Hunayn », $E P^{2}$, p. ****.

SUERMANN H.

2009 «John the Stylite of Mār Z urā at Sarug », dans Christian-Muslim relations. 1, 600-1500, ed. by D. Thomas \& B. Roggema, Leiden, p. ***

TAKAHASHI H.

2011 «Bar `Ebroyo, Grigorios », GEDSH, p. 54-56.

TALMON R.

2000 «Foreign influence in the Syriac grammatical tradition ", dans History of the language sciences. 1, ed. by S. Auroux et al., Berlin - New York, p. 337-341.

2008 « Jacob of Edessa the Grammarian », dans Jacob of Edessa and the culture of his day, ed. by B. ter HAAR RoMenY, Leiden, p. 159-187.
TLG Thesaurus Linguae Graecae (TLG) : a digital library of Greek literature, Irvine, 2001.

Vosté J.

1929 Catalogue de la bibliothèque syro-chaldéenne du couvent de Notre-Dame-des-Semences près d'Alqoš (Iraq), Rome - Paris.

WATT J. W.

1993 « Grammar, rhetoric and enkyklios paideia in Syriac», Zeitschriften der Deutschen Morgenländischen Gesellschaft 143, p. 45-71.

2004 « Syriac translators and Greek philosophy in early Abbasid Iraq ", Journal of the Canadian society for Syriac studies 4, p. 15-26.

WRIGHT W.

1871 Fragments of the Turras mamlla nahraya or Syriac Grammar of Jacob of Edessa, London. 
